\section{MS6-04 General mechanism of function of TIR domains}

Bostjan Kobe ${ }^{1}$, Thomas Ve ${ }^{1}$, Simon Williams ${ }^{1}$, Xiaoxiao Zhang ${ }^{1}$, Li Wan ${ }^{1}$, Mohammed Alaidarous ${ }^{1}$, Maud Bernoux ${ }^{2}$, Kee Hoon Sohn $^{3}$, Jonathan Jones ${ }^{3}$, Michael Landsberg ${ }^{1}$, Peter Dodds ${ }^{2}$

1. University of Queensland, Brisbane, Australia

2. CSIRO, Agriculture Flagship, Canberra, Australia

3. The Sainsbury Laboratory, Norwich, UK

email: b.kobe@uq.edu.au

TIR (Toll/interleukin-1 receptor, resistance protein) domains are key components of innate immunity signalling pathways. They are found in animals, plants and bacteria, for example in TLRs (Toll-like receptors) and TLR adaptors in animals, NLRs (nucleotide binding, leucine-rich repeat) in plants, and virulence factors interfering with immune responses in bacteria. While it has been well established that signalling depends on regulated self-association and homotypic association of TIR domains, every single TIR domain structure has revealed a different association mode [1]. In the search for common features, we have targeted a number of TIR domains from mammals, plants and bacteria to characterize structurally. We have determined the crystal structures of the TIR domains from the human TLR adaptor protein MAL [2], the bacterial protein TcpB from Brucella melitensis [3] and the plant immune proteins L6 from flax [4], RPS4 and RRS1 from Arabidopsis [5], SNC1 from Arabidopsis and MrRUN1 and MrRPV1 from grapevine (unpublished). In the case of the proteins RPS4 and RRS1, which work together as a protein complex to confer resistance to three different bacterial and fungal pathogens, we have determined, using linker-assisted crystallization, the first structure of a hetero-dimeric complex of TIR domains. The association interface in this complex is conserved in the crystals of the TIR domains of RPS4 and RRS1 on their own, as well as in those of SNC1, MrRUN1 and MrRPV1. Similarly, the dimerization interface observed in the structure of TcpB is conserved in the structure of the TIR domain-containing protein from Paracoccus denitrificans. We further observed that TLR4 TIR domains seed helical filament formation by MAL (cryo-electron microscopy reconstruction shown in Fig. 1), which in turn seeds a crystalline assembly of the TIR domain from TLR adaptor MyD88 (the crystals are currently being characterized by X-FEL). Jointly, these studies are yielding a general mechanism of function of TIR domains, which at least in some cases will involve signaling through higher-order assembly formation with prion-like features.[1] Ve T, Williams S, Kobe B (2015) Apoptosis 20: 250-61 [2] Valkov E et al, Proc Natl Acad Sci USA, 2011, 108, 14879-14884 [3] Alaidarous M et al, J Biol Chem, 2014, 289, 654-68 [4] Bernoux M et al, Cell Host Microbe, 2011, 9, 200-211 [5] Williams SJet al, 2014, Science 344: 299-30
A

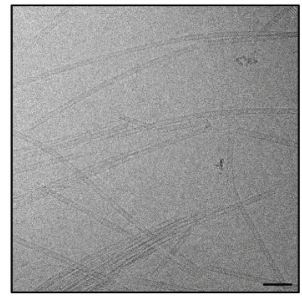

B

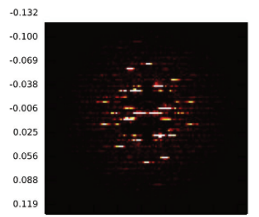

E

$\mathrm{F}$
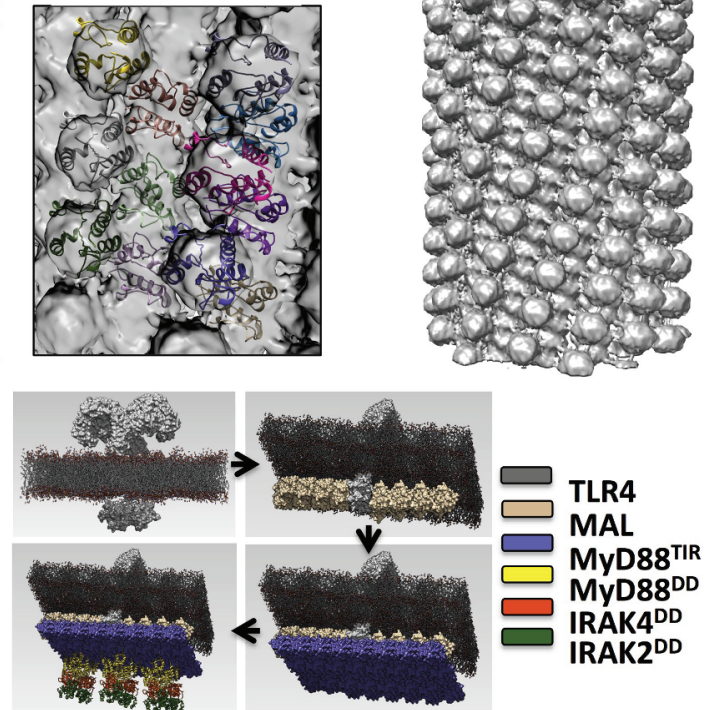

Figure 1. Structure of MAL filaments. A. Cryo-EM image. B. Power spectrum from a class average. The layer lines indicate helical symmetry. C/D. Cryo-EM reconstruction. E. Zoom-in showing MAL monomers fitted into electron density. F. Working model for signaling by TLR4/MAL/MyD88.

Keywords: Innate immunity, Bacterial pathogenesis, Linker-assisted crystallization, Cryo-electron microscopy, X-ray free-electron laser 